\title{
Delayed Onset Contralateral Reexpansion Pulmonary Edema after Tension Pneumothorax
}

\author{
- A Case Report - \\ Dongseop Song, M.D. and Jai Yun Jung, M.D.* \\ Departments of Thoracic and Cardiovascular Surgery, *Anesthesiology and Pain Medicine, Sanbon Hospital, \\ College of Medicine, Wonkwang University, Gunpo, Korea
}

\begin{abstract}
A 16-year-old male patient presented with left side chest pain. The initial chest radiograph showed tension pneumohtorax on the left side. Air was evacuated by closed thoracostomy. About 72 hours later, during administration of general anesthesia for thoracoscopic bullectomy, unilateral pulmonary edema affecting the contralateral lung developed without definite infiltration in the left lung. The operation was suspended and the patient was admitted to the intensive care unit. A close observation of the patient and conservative therapy were enough to manage this pulmonary edema. This is a very rare manifestation of reexpansion pulmonary edema that is unpredictable and could be fatal. The clinical course is described in this article.
\end{abstract}

Key Words: contralateral, pneumothorax, pulmonary edema.

Reexpansion pulmonary edema (RPE) is a rare complication that occurs after the expansion of collapsed lung. Moreover, contralateral RPE without ipsilateral lung involvement is extremely rare. We experienced contralateral RPE in a spontaneous tension pneumothorax patient in a unique situation. The first symptom presentation of RPE was in the middle of general anesthesia and it was about 72 hours after lung expansion. The pathophysiology of RPE is not clearly understood yet, so this case might be a good reference to review RPE mechanisms.

\section{CASE REPORT}

A 16-year-old male without significant medical history was admitted to our emergency department. He complained left side chest pain and shortness of breath existing for 1 day. The ini-

Received on November 7, 2012, Revised on January 24, 2013, Accepted on February 17, 2013

Correspondence to : Dongseop Song, Department of Thoracic and Cardiovascular Surgery, Sanbon Hospital, Wonkwang University, 1142, Sanbon-dong, Gunpo 435-040, Korea

Tel: 031-390-2214, Fax: 031-390-2999

E-mail: csman2002@hanmail.net

In this paper, the research was supported by Wonkwang University 2011 research fund program. tially checked chest radiograph revealed a totally collapsed left lung which suggested spontaneous tension pneumothorax (Fig. 1A). Urgent thoracostomy was performed with a 24 French chest tube. Massive air was evacuated and the chest tube was connected to natural drainage system (under water seal bottle). The following chest radiograph showed fully expanded lungs (Fig. 1B). High resolution computed tomography was obtained one hour after thoracostomy and it presented two bullae over the apical area of his left lung and minimal infiltration of his left lower lobe within the subsegmental area (Fig. 2). The patient was then admitted to general ward. The chest radiograph checked on the 2nd and 3rd hospital day showed no lung infiltration or other significant change. Air leakage continued for 4 days. On his 4th hospital day, a thoracoscopic bullectomy was scheduled. The patient's height and body weight were 174 $\mathrm{cm} / 52 \mathrm{~kg}$. Preoperatively checked laboratory findings were absolutely within normal range. The white blood cell count (WBC) was 9,100/ $/ 1$, and the hemoglobin was $15.6 \mathrm{~g} / \mathrm{dl}$. ALT was $10.0 \mathrm{IU} / \mathrm{L}$ and AST was $15.0 \mathrm{IU} / \mathrm{L}$. Total protein and albumin were $6.7 \mathrm{~g} / \mathrm{dl}$ and $4.0 \mathrm{~g} / \mathrm{dl}$ in each. The electrolytes level showed $142 \mathrm{mmol} / \mathrm{L}$ of sodium, $3.6 \mathrm{mmol} / \mathrm{L}$ of potassium and $103 \mathrm{mmol} / \mathrm{L}$ of chloride. EKG finding showed no abnormality. The patient underwent total intravenous anesthesia 

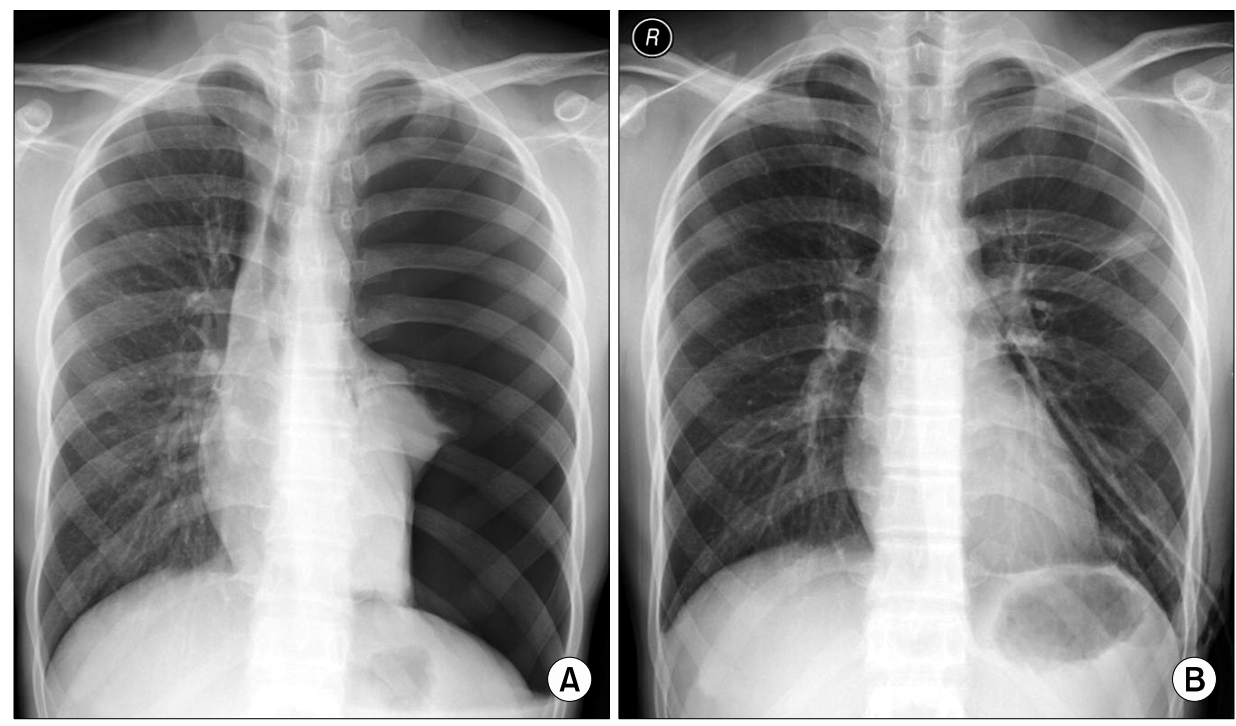

Fig. 1. (A) The initial chest PA shows tension pneumothorax on left. (B) Immediately after thoracostomy, the left lung has expanded and both lung fields have no infiltration.

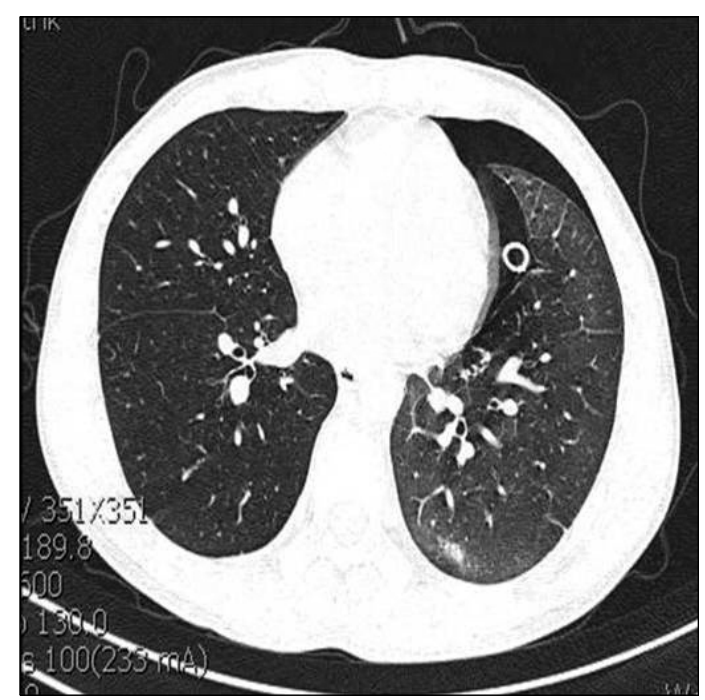

Fig. 2. HRCT shows minimal infiltration on left lower lobe.

with propofol and remifentanil. Target-controlled infusion started with initial effect site concentration of $4 \mu \mathrm{g} / \mathrm{ml}$ for propofol and $4 \mathrm{ng} / \mathrm{ml}$ for remifentanil respectively. After loss of consciousness the patient were intubated with double lumen tube after rocuronium $0.6 \mathrm{mg} / \mathrm{kg}$ intravenous injection. The location and function of the intubation tube were verified under bronchoscopic exam. And the patient was settled on the right decubitus position. After routine preparation, right one lung ventilation started by clamping his left bronchial tube. The peak inspiratory pressure increased up to $45 \mathrm{mmHg}$. The pulse oximetry revealed an oxygen saturation level below 60\%. The end tidal $\mathrm{CO}_{2}$ was above $41 \mathrm{mmHg}$. We repeated two lung ventilation and one lung ventilation several times with careful air way toileting and determined that the patient could not bear one lung ventilation. A left side chest tube was re-inserted with a 28 French chest tube. And the patient's position was changed into supine position. A portable chest radiograph checked at the operating room (OR) showed complete right lung infiltration (Fig. 3A). With two lung ventilation the patient's respiration was stable. Small dosage of furosemide (10 $\mathrm{mg}$ once at OR) was administered. The patient was extubated at the OR and referred to intensive care unit (ICU). At the OR $600 \mathrm{ml}$ of Hartmann solution was infused and his urine output was $750 \mathrm{ml}$. Under close observation on his respiration, volume restriction was maintained at ICU. The arterial blood chemistry showed $47 \mathrm{mmHg}$ of $\mathrm{PCO}_{2}$ and $86 \mathrm{mmHg}$ of $\mathrm{PO}_{2}$ in room air. But the patient showed no tachypnea or shortness of breath. The WBC count after operation was $10,100 / \mu 1$ and the CRP was $2.0 \mathrm{mg} / \mathrm{dl}$. First generation cephalosporin was used throughout his hospital course. But we did not change or add the antibiotics at all. Serial chest radiographs showed improvement and the respiration was stable in room air. The patient was referred to the general ward in the evening of operation day. The chest radiograph obtained in the next morning showed a recovered right lung (Fig. 3B). And then there was no further event. The air leakage continued and a 2nd operation for thoracoscopic bullectomy was undertaken on the 9th hospital day. The chest tube was removed on the 12th hospital day and the patient was discharged on that day.

\section{DISCUSSION}

RPE was first described in 1853 by Pinault.[1] He men- 

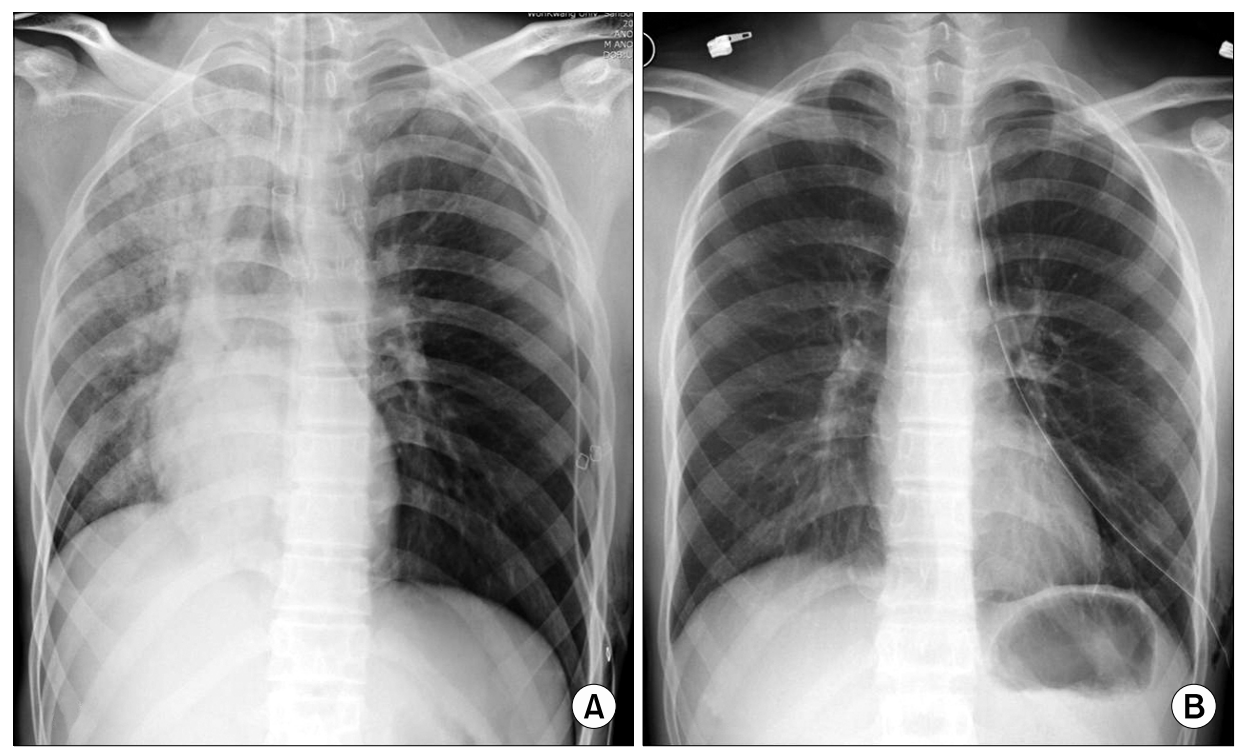

Fig. 3. (A) A portable chest radiograph checked at the operating room shows complete right lung infiltration. (B) The chest radiograph obtained next morning shows a recovered right lung.

tioned about pulmonary edema occurring in a reexpanded lung after the removal of pleural effusion. Recently, the most famous predisposing condition of RPE is pneumothorax. RPE is considered to take place after sudden expansion of a chronically collapsed lung for more than 3 days, but it can also develop in lungs with short collapse duration. The incidence of RPE is as low as 0.5\%.[2] In general, the clinical course of RPE is brief and controlled well with diuretics or steroids. Also, RPE could be a potentially fatal complication, especially when it involves bilateral lungs and the mortality reaches up to $20 \%$. RPE generally develops immediate after lung expansion but it can also develop within 48 hours after lung expansion.[3]

There are several possible explanations for the development of RPE. 1) Mechanical injury of pulmonary vascular endothelium, 2) Surfactant deficit due to chronic collapse of lung, 3) Oxygen free radical injury originated by ischemia-reperfusion and 4) PMN cell related inflammation cascade.[4] Sudden evacuation of fluid or air might be considered as promoting events. But, any of these theories cannot explain contralateral RPE without ipsilateral RPE.

Unilateral pulmonary edema can develop in several specific conditions such as unilateral air way obstruction, injured lung, pulmonary vascular disease or prolonged decubitus positioning. In this case, the patient was previously healthy without any particular medical history. Preoperative examination showed no cardiopulmonary abnormality, hepatic, or renal insufficiency. As mentioned before, there was no volume overloading during anesthesia and we could not find out the relationship between the brief ducubitus position and right side edema. Most of all, the brief clinical course and rapid response to conservative treatment demonstrates that this is a RPE.

The first video assisted thoracic surgery exploration underwent on the 4th hospital day. It was about 72 hours after thoracostomy. Although the collapsed left lung showed minimal infiltration immediately after thoracostomy, a chest PA on the day before the operation showed fully expanded normal lungs. Mahfood et al.[3] reported that onset of RPE following pneumothorax is almost within 1 hour and in all cases the onset occurred within 24 hours. And as mentioned before, RPE generally develops within 48 hours after lung expansion.

RPE involving contralateral lungs is rare but reported on occasion. In 1987, Mahfood et al.[3] reviewed 47 RPE case reports following pneumothorax and there were only 2 contralateral RPE cases, which took place immediately after thoracostomy. In other reports, contralateral RPE were presented with ipsilateral RPE. Contralateral RPE without ipsilateral RPE is extremely rare, especially 72 hours after lung expansion.

Cohen et al.[5] reported a case of contralateral RPE following unilateral transaxillary sympathectomy. This patient showed right side RPE immediately after left sympathectomy. The author concluded that the sympathectomy could protect the left lung from RPE. Heller and Grathwohl[6] described left side RPE following right pleural fluid drainage of a liver cirrhosis patient. They assumed that the intrapulmonary shunting from hepatopulmonary syndrome was potentially worsened, causing hypoxemia and uneven pulmonary blood flow to the left lung. There are other explanations for the mechanism of contralateral 
RPE without ipsilateral RPE. In a lung-injured patient, if normal tidal volume $(10 \mathrm{ml} / \mathrm{kg})$ under general anesthesia were used, the entire volume could be transmitted to the healthy lung. That could be the reason for subclinical barotrauma of healthy lung and cause RPE.[7] Chang et al.[8] described the animal model that explains barotrauma of the healthy lung under one lung ventilation over $60 \mathrm{~min}$. Sohara[4] insisted that in pneumothorax patients, compression atelectasis of the contralateral lung associated with the shift of the mediastinum was believed to be the reason of contralateral RPE.

In this case, the patient has no specific underlying disease and there was no volume overloading. And general anesthesia was continued for less than $30 \mathrm{~min}$ since tracheal intubation. As soon as the inspiration peak flow increased, the one lung ventilation was abandoned. In conclusion, contralateral RPE without definite ipsilateral RPE developed 72 hours after lung expansion. This situation was very unpredictable and could be lethal. It's considered as a very rare presentation of RPE. The pathophysiology of RPE is not clearly understood yet, so this case might be a good reference for pursuing studies in RPE mechanisms.

\section{REFERENCES}

1) Riesman D: Albuminous expectoration following thoracentesis. Am J Med Sci 1902; 123: 620-30.

2) Feller-Kopman D, Berkowitz D, Boiselle P, Ernst A: Largevolume thoracentesis and the risk of reexpansion pulmonary edema. Ann Thorac Surg 2007; 84: 1656-61.

3) Mahfood S, Hix WR, Aaron BL, Blaes P, Watson DC: Reexpansion pulmonary edema. Ann Thorac Surg 1988; 45: 340-5.

4) Sohara Y: Reexpansion pulmonary edema. Ann Thorac Cardiovasc Surg 2008; 14: 205-9.

5) Cohen JD, Loewinger J, Zelikovski A, Gassner S: Unilateral pulmonary oedema of the contralateral lung following transaxillary sympathectomy. Anaesthesia 1991; 46: 651-3.

6) Heller BJ, Grathwohl MK: Contralateral reexpansion pulmonary edema. South Med J 2000; 93: 828-31.

7) Gordon AH, Grant GP, Kaul SK: Reexpansion pulmonary edema after resolution of tension pneumothorax in the contralateral lung of a previously lung injured patient. J Clin Anesth 2004; 16: 289-92.

8) Chang CY, Hung MH, Chang HC, Chan KC, Chen HY, Fan SZ, et al: Delayed onset of contralateral pulmonary edema following reexpansion pulmonary edema of a collapsed lung after video-assisted thoracoscopic surgery. Acta Anaesthesiol Taiwan 2009; 47: 87-91. 